\title{
Flexible Emergency Vehicle Network Design considering Stochastic Demands and Inverse-Direction Lanes
}

\author{
Hua Wang $\mathbb{D}^{1,2}$ Ling Xiao, ${ }^{1}$ and Zhang Chen $\mathbb{D}^{3}$ \\ ${ }^{1}$ School of Economics and Management, Tongji University, Shanghai 200092, China \\ ${ }^{2}$ Department of Civil \& Environmental Engineering, National University of Singapore, Singapore 117576 \\ ${ }^{3}$ Key Laboratory of Road and Traffic Engineering of the Ministry of Education, Tongji University, Shanghai 200092, China \\ Correspondence should be addressed to Zhang Chen; czy1620@263.net
}

Received 10 December 2017; Accepted 1 February 2018; Published 8 March 2018

Academic Editor: Xiaobo Qu

Copyright (c) 2018 Hua Wang et al. This is an open access article distributed under the Creative Commons Attribution License, which permits unrestricted use, distribution, and reproduction in any medium, provided the original work is properly cited.

We study transportation network design with stochastic demands and emergency vehicle (EV) lanes. Different from previous studies, this paper considers two groups of users, auto and EV travelers, whose road access rights are differentiated in the network, and addresses the value of incorporating inverse-direction lanes in network design. We formulate the problem as a bilevel optimization model, where the upper-level model aims to determine the optimal design of EV lanes and the lower-level model uses the user equilibrium principle to forecast the route choice of road users. A simulation-based genetic algorithm is proposed to solve the model. With numerical experiments, we demonstrate the value of deploying inverse-direction EV lanes and the computational efficiency of the proposed algorithm. We reach an intriguing finding that both regular and EV lane users can benefit from building EV lanes.

\section{Introduction}

Emergency incidents, such as traffic accidents, mere spasmodic diseases, and fire disasters, have often been observed in metropolises with a high population density $[1,2]$, which generates an increasing travel demand of emergency vehicles (EVs) (e.g., ambulances and fire engines). Currently, transportation emergency evacuation and rescue have attracted greater attention from transportation researchers and practitioners than before. For example, So and Daganzo [3] proposed an inner-first-out control strategy to manage evacuation routes, and Daganzo and So [4] extended such a nonanticipative and adaptive control strategy to manage traffic networks in real-time emergency evacuations. Meanwhile, with advances in intelligent transport techniques, fast development in building various emergency evacuation systems is witnessed, for example, the corridor-based and region-wide emergency evacuation systems for Washington D.C. [5] and Baltimore's multimodal evacuation system [6]. In addition, the Transportation Research Board [7] made a tentative investigation to accommodate emergency evacuation into transit service systems.
Effective strategic-level or tactical-level planning is one of the important prerequisites to ensure the success of operational-level emergency evacuation management [8]. For example, transportation authorities often reserve proper emergency lanes on highway networks to promote travel efficiency of emergency vehicles in accident rescues. As a result, a few interesting questions arise. Can we design similar emergency lanes in urban transportation networks? Also, how can we achieve successful planning of emergency lanes in urban transportation systems with limited road infrastructure resources? Moreover, complex traffic conditions in urban transportation networks, such as demand uncertainties and complicated user behaviors, lead to new challenges for the planning of emergency lanes $[9,10]$. In this study, we aim to present feasible answers from the perspective of transportation network design. A running example is presented as follows to illustrate the problem of designing emergency vehicle (EV) lanes.

1.1. Running Example. Figure 1(a) shows a small urban transportation network with three nodes and five directed links. Link 1 has two lanes and each of links $2-5$ is deployed with 


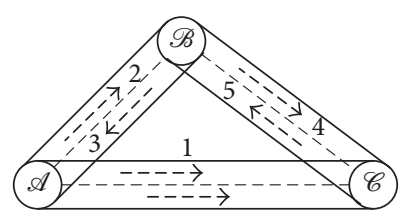

(a) Original transportation network

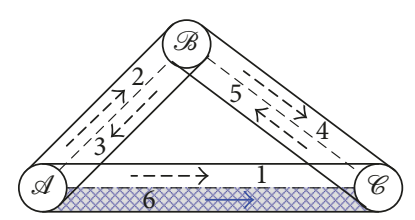

(b) New network with an EV lane

FIGURE 1: A three-node network for the running example.

TABLE 1: Results of three design schemes in the running example.

\begin{tabular}{|c|c|c|c|c|c|c|c|c|}
\hline \multirow{2}{*}{ Design schemes } & \multicolumn{6}{|c|}{ Equilibrium link flows } & \multirow{2}{*}{ TTT for auto users (mins) } & \multirow{2}{*}{ TTT for EV users (mins) } \\
\hline & $v_{1}$ & $v_{2}$ & $v_{3}$ & $v_{4}$ & $v_{5}$ & $v_{6}$ & & \\
\hline Scheme 1 & $26600 / 17$ & $8250 / 17$ & 1060 & $8250 / 17$ & 1060 & - & $60,367.06$ & $2,374.38$ \\
\hline Scheme 2 & $13000 / 11$ & $9000 / 11$ & 1060 & $9000 / 11$ & 1060 & 50 & $68,356.36$ & $2,008.20$ \\
\hline Scheme 3 & $13300 / 11$ & $9250 / 11$ & 1000 & $9250 / 11$ & 1000 & 60 & $68,181.82$ & $1,740.55$ \\
\hline
\end{tabular}

a respective single lane. All lanes have an identical service capacity of 1,000 vehicles/hour. Two origin-destination (OD) pairs, $\mathscr{A} \rightarrow \mathscr{C}$ and $\mathscr{C} \rightarrow \mathscr{A}$, are taken into account. There exist two classes of users, auto commuters and EV travelers, in the network. Both classes of network users are assumed to follow Wardrop's first equilibrium principle that each of the road users expects to find her/his shortest path. The auto travel demands for two OD pairs are, respectively, $q_{\mathscr{A} \rightarrow \mathscr{C}}^{p}=$ 2,000 and $q_{\mathscr{C} \rightarrow \mathscr{A}}^{p}=1,000$. The EV travel demands for two OD pairs are set as $q_{\mathscr{A} \rightarrow \mathscr{C}}^{e}=50$ and $q_{\mathscr{C} \rightarrow \mathscr{A}}^{e}=60$. To improve the travel efficiency of EVs, the network authority attempts to build an EV lane on Link 1. As shown in Figure 1(b), the original Link 1 with two lanes would be then decomposed into a new regular road with one lane (Link 1) and a new $\mathrm{EV}$ road with one lane (Link 6). Let us define a link set $A:=$ $\{1,2, \ldots, 6\}$. A linear link travel time function is adopted here to evaluate the network performance:

$$
t_{a}\left(v_{a}^{p}, v_{a}^{e}\right)=t_{a}^{0}+t_{a}^{0}\left(\frac{v_{a}^{p}+v_{a}^{e}}{c_{a}}\right), \quad a \in A,
$$

where $t_{a}^{0}$ is the flow-independent travel time of link $a, a \in A$; $v_{a}^{p}$ and $v_{a}^{e}$, respectively, represent link flows of auto users and EV travelers; and the aggregate link flow $v_{a}=v_{a}^{p}+v_{a}^{e}$. In this example, we set $t_{2}^{0}=t_{3}^{0}=5 \mathrm{mins}, t_{4}^{0}=t_{5}^{0}=7 \mathrm{mins}$, and $t_{1}^{0}=t_{6}^{0}=10$ mins.

We consider and compare the following three EV lane design schemes.

Scheme 1. Take no action of setting EV lanes.

Scheme 2. Build an EV lane to connect the nodes $\mathscr{A}$ and $\mathscr{C}$ (Link 6 in Figure 1).

Scheme 3. Build an inverse-direction EV lane to access $\mathscr{C} \rightarrow$ $\mathscr{A}$ by reversing the direction of Link 6 .

Table 1 gives the aggregate link flows at biclass user equilibrium and total travel time (TTT) for auto and EV users.
As shown in Table 1, Scheme 2 sets an EV link and saves about $15.4 \%$ golden rescue time, although it makes auto travelers suffer uncomfortable experiences in a more congested network. Compared to Scheme 2, Scheme 3 not only remarkably improves the travel efficiency of EVs but also alleviates the traffic congestion of auto commuters through flexibly incorporating an inverse-direction EV lane. In this sense, Scheme 3 better balances the two design objectives of TTTs associated with auto and EV users. The results of network performance improvements clearly demonstrate the significance of building EV lanes and reveal the value of designing inversedirection EV lanes in EV network planning.

The running example clearly shows that the investigated problem of setting EV lanes might fall into the category of classical discrete transportation network design problems (NDPs), which can be defined as a new link/lane addition that maximizes network performance subject to a series of side constraints (e.g., investment budget constraint and equilibrium constraints of the traffic pattern that characterizes travelers' route choice behaviors). However, the NDP that optimizes EV lane setting (EV-NDP) also exhibits a few new features and disparities from traditional NDPs (explained later in the next subsection). This study is devoted to proposing an effective approach for the EV-NDP that accounts for more complex and realistic traffic conditions, including application of flexible inverse-direction EV lanes and travel demand uncertainties.

1.2. Literature Review. The NDP typically aims to optimize transportation network performance via link capacity expansions (either current road maintenance/improvement or new road addition), while taking into account travelers' route choice behaviors and financial constraints. After the seminal works of Leblanc [11] and Abdulaal and LeBlanc [12], we have witnessed a blooming of NDPs on model formulations, solution algorithms, and applications over the past half century. Regarding early achievements made in the last century, interested readers are referred to two surveys, Magnanti and Wong [13] and Yang and Bell [14], for a 
comprehensive literature review and detailed discussions. Meng and Wang [15] presented a structured overview of the NDP literature from 1999 onward and summarized a series of new developments and advances that consider complicated traffic conditions, including uncertainty parameters, traffic dynamics, and multiple optimization objectives. We briefly review the recent achievements of the NDP literature in the following four NDP categories.

(1) Stochastic NDPs. It is well recognized that network uncertainties with respect to demand fluctuations and road capacity degradation inevitably and inherently exist on transportation networks. Recently, more researchers and practitioners have pointed out that network uncertainty would have significant impacts on network planning and other transportation management decisions, and ignoring this factor might result in suboptimal or even misleading optimization schemes $[8,16,17]$. Chen et al. [18] provided a comprehensive review of stochastic NDPs on various model formulations (e.g., expected-value model, mean-variance model, chance-constrained model, probability model, and min-max model) and proposed an attractive simulationbased genetic algorithm. To properly characterize the effect of network stochasticity, simulation-based sampling (e.g., Monte-Carlo simulation) approximation technique is often used to estimate probabilistic statistics, including expectation and variance, of various system performance measures $[8,18-24]$. Alternatively, probabilistic or reliability-based user equilibriums are proposed to incorporate the effect of network uncertainties into travelers' route choice behaviors in the lower-level traffic assignment problem and similar bilevel stochastic NDP models are then developed $[25,26]$. Some stochastic NDP models had also integrated other traffic factors and/or optimization targets (e.g., sustainability, traffic dynamics, and land use). For example, Waller and Ziliaskopoulos [16] formulated a chance-constrained NDP model that takes into account both uncertain demands and dynamic traffic flows. Wang et al. [26] incorporated multiple indices of sustainability, including traffic emission and social equity, into a stochastic NDP.

(2) Dynamic and Multiperiod NDPs. Time dimension is another important factor in transportation network design. In general, three scales of time dimension are considered: short-term/real-time (seconds), medium-term (days), and long-term (years). Overall, short-term traffic dynamics are attributed to the description of real-time parameter fluctuation in a transportation system, and long-term network decisions across multiple phases help to assess the value of a network improvement plan over time. Specifically, dynamic NDP models have been developed to properly describe realtime traffic dynamics and/or other unsteady-state traffic conditions (e.g., traffic shockwaves propagation, build-up process of queues) [27]. For example, Waller and Ziliaskopoulos [16] proposed a two-stage linear programming NDP model where a system-optimal dynamic traffic assignment and cell transmission loading technique were used to depict dynamic traffic propagation on the network. Karoonsoontawong and Waller [27] presented a robust dynamic NDP model with the objective of minimizing the weighted sum of the expected total travel time and expected risk. In detail, multiperiod NDP formulations were proposed to optimize a design scheme where construction and maintenance of transport infrastructures took place over a long-term horizon. Lo and Szeto [28] proposed the first multiperiod NDP model to examine tradeoffs among several stakeholders: travelers, private toll road operators, and the government. Ukkusuri and Patil [29] made an extension to formulate a multiperiod NDP considering demand uncertainty and demand elasticity, and they found that a multiperiod optimization model could generate more favorable decisions than the conventional single-stage NDP formulation. A more comprehensive literature review and detailed discussions on dynamic and multiperiod NDPs can be found in Meng and Wang [15].

(3) Multiobjective NDPs. As reported by Yang and Bell [14] and Chen et al. [20], researchers often need to consider multiple design objectives in the NDP, for instance, travel efficiency, emission, social equity, user experience, construction cost, and system reliability. There is a great deal of literature on multiobjective NDPs. For example, Meng and Yang [30] discussed a biobjective NDP regarding travel efficiency and social equity, and Chen and Yang [23] made an extension to analyze the NDP incorporating social equity issue in stochastic networks and developed a chance-constrained optimization model. Wang et al. [26] investigated a chanceconstrained multiobjective NDP considering social equity, emission reduction, and network reserve capacity maximization. In general, Pareto optimization method (e.g., [19-21, 31]) and weighted-sum approach (e.g., $[8,22])$ are widely applied for multiobjective NDP formulations. For more information on deterministic multiobjective NDPs, refer to review reports of Yang and Bell [14] and Farahani et al. [32], and more literature on stochastic multiobjective NDPs can be found in Chen et al. [18].

(4) Global Solution Methods. Solving a bilevel NDP model has been seen as a challenge all the time. Global solution algorithms have increasingly received attention in the last decade. For example, based on the Multivariate Taylor Series, Wang and Lo [33] transformed the classical NDP model into equivalent mixed-integer linear programming (MILP), in which a global-optimal solution can be guaranteed. In spirit of the transformed MILP, Luathep et al. [34] made an extension to solve a mixed NDP with both continuous and discrete decision variables. Motivated by a similar idea, Liu and Wang [35] developed an equivalent MILP model for the NDP that took into account logit-based stochastic user equilibrium. The above-mentioned MILP models were all solved by the CPLEX solver. In addition, according to the relationship between user equilibrium and system optimum, Wang et al. [36] presented a system optimum-relaxation based method to solve the discrete NDPs.

The EV-NDP in this study exhibits some new features and differs from existing NDPs in the following aspects:

(1) The investigated EV-NDP involves two classes of users: auto commuters and EV travelers. The above 
literature review indicates that the issue of multiclass users has received rather limited attention. The only NDP work that considered multiclass users was carried out by Dimitriou et al. [37]. Incorporating multiclass users leads to challenges in two aspects, especially when considering uncertain demands: (i) the lower-level traffic assignment problem needs to characterize route choice behaviors of multiple classes of users and resultant equilibrium traffic patterns may not be unique and (ii) the presence of multiclass users probably gives rise to conflict design objectives. It is indeed a challenge to manage tradeoffs among different design objectives associated with multiple classes of users on stochastic networks.

(2) The EV-NDP has a different network reconstruction principle from traditional discrete NDPs. Traditional discrete NDP determines link/lane additions to expand network capacities [14]. The investment is directed to improve link capacity, while network structure accessibility (i.e., topological structure) might keep unchanged. However, in EV-NDP, the total capacity remains unchanged, but certain capacity is removed from original regular links to form new EV links (hereafter, EV links are called derivative links). Take the case in Figure 1 as an example. EV Link 6 is a derivative of Link 1 , and setting the EV link results in a capacity reduction of the original Link 1. This can be regarded as link decomposition and capacity reassignment/redeployment. For an EVNDP scheme, the construction budget is invested to build supporting infrastructures (e.g., pilot signals, guide boards, and communication facilities) that control and manage various vehicles, rather than directly expanding link capacities.

(3) Road access rights are differentiated for two classes of users. As mentioned above, conventional NDPs consider homogeneous commuters and every traveler can freely utilize any link if accessible. This condition is also satisfied in Dimitriou et al. [37], in which multiclass users are taken into account. However, the situation in EV-NDPs differs. EVs can freely use all roads without any restraints, but private cars are not allowed to run on EV lanes. Such differentiated road access rights lead to a challenge in formulating a biclass user equilibrium that describes different travelers' route choice behaviors.

(4) Inverse-direction EV lanes are introduced for EVNDPs to fulfill favorable decision schemes. Given the fact that EV trips are independently and separably regulated by the traffic control center during emergency periods, we could have a chance to set inversedirection EV lanes. This interesting concept makes network design scheme more flexible and attractive.

With the above stated disparities, we need to develop new EV-NDP models and propose pertinent solution methods.
1.3. Objective and Contributions. The objective of this paper is to propose an effective approach for the EV-NDP with demand uncertainty. To achieve this objective, we firstly propose a network reconstruction method to build a new unified network where both auto and EV users are properly accommodated. We then develop a bilevel optimization model for the EV-NDP on stochastic networks, where the upper level aims to minimize the weighted sum of the expected total travel time of two classes of users and the lower level characterizes route choice behaviors of auto and EV travelers under each demand realization.

This study makes the following substantial contributions to the literature:

(1) Our work investigates a new NDP in urban transportation networks that exhibit several disparities from traditional NDPs. To the best of our knowledge, this work addresses a new EV network planning problem and is the first to formulate NDP with multiclass users, which substantially bridges the gap of the existing literature.

(2) We develop a bilevel optimization EV-NDP model considering uncertain demands to find the tradeoff between travel efficiencies for auto and EV travelers. The proposed methods can help transportation authorities make tangible EV network decisions.

(3) We introduce a new concept of inverse-direction EV lane in the EV-NDP. Incorporating inverse-direction EV lanes could promote more flexible and attractive decisions.

(4) We propose a simulation-based genetic algorithm (GA) to solve the developed EV-NDP model and further evaluate the model and computational efficiency of the solution algorithm through numerical experiments. The evaluation results help us better understand the importance of considering inversedirection EV lanes in EV network planning and the value of simulation-based GA in solving a bilevel stochastic programming model.

With all the above explanations, we shall devote Section 2 to defining the problem and formulating it as a bilevel optimization model. We then present in Section 3 a simulation-based genetic algorithm to solve the EV-NDP model. Numerical examples are provided in Section 4. The final section, Section 5, concludes the paper.

\section{Problem Description and Model Formulation}

We first define in this section the EV-NDP. Then, we explain the network reconstruction and formulate the network design model of setting EV lanes (the EV-NDP model).

2.1. Problem Description. Consider an urban transportation network $G=(N, A)$, where $N$ and $A$ are the sets of nodes and links, respectively. There exist two classes of users in the network: auto travelers and EV users. To guarantee the travel 
efficiency of EVs, the network authority designs an EV-NDP scheme that deploys exclusive EV lanes on the selected roads. To better evaluate the network performance, we take into account random travel demands for both auto and EV users.

The network planner not only aims to guarantee the travel efficiency of EVs, but also makes an attempt to improve the travel efficiency of auto travelers. The design objective function is thus to minimize the weighted sum of the expected total travel time of two classes of users on the network. Let $\bar{A}$ denote the set of candidate links to be deployed with EV lanes. The decision variables can be defined as

$$
\begin{aligned}
& \widehat{x}_{a}:=\left\{\begin{array}{ll}
1 & \text { if an EV lane in the same direction of link } a \text { is deployed } \\
0 & \text { otherwise }
\end{array} \forall a \in \bar{A},\right. \\
& \tilde{x}_{a}:=\left\{\begin{array}{ll}
1 & \text { if an EV lane in the inverse direction of link } a \text { is deployed } \\
0 & \text { otherwise }
\end{array} a \in \bar{A} .\right.
\end{aligned}
$$

2.2. Notations and Assumptions. The following notations are used throughout the paper.

$N$ : the set of nodes in a given urban transportation network $G$;

$A$ : the set of original links in the urban transportation network $G$;

$\bar{A}$ : the set of candidate physical links considered in the EV-NDP scheme, $\bar{A} \subseteq A$;

$\widehat{A}$ : the set of normal-direction EV links, $\widehat{A} \subseteq A,|\widehat{A}|=$ $|\bar{A}|$;

$\widetilde{A}$ : the set of inverse-direction EV links, $\widetilde{A} \subseteq A,|\widetilde{A}|=$ $|\bar{A}|$;

$A^{\prime}$ : the set of links in the reconstructed unified network, $A^{\prime}:=A \cup \widehat{A} \cup \widetilde{A}$;

$W$ : the set of OD pairs in the transportation network;

$K$ : the set of user classes where $K:=\{p, e\}$, where $p$ and $e$ represent private car and emergency vehicle, respectively;

$R_{w}^{k}$ : the set of travel paths associated with $k \mathrm{th}, k \in K$ users and OD pair $w \in W$;

$\Omega$ : the set of demand realizations;

$\omega$ : a specific demand realization $\omega:=\left(\omega^{p}, \omega^{e}\right) \in \Omega$;

$S$ : the set of scenarios to realize the random travel demands;

$v_{a}^{k, \omega}:$ traffic flow of $k$ th, $k \in K$ users on link $a$ under demand realization $\omega \in \Omega$;

$v_{a}^{\infty}$ : the aggregate traffic flow on link $a$ under demand realization $\omega \in \Omega$;

$f_{r, w}^{k, \omega}:$ traffic flow of $k$ th, $k \in K$ users on travel route $r, r \in R_{w}^{p}$ for OD pair $w, w \in W$ under demand realization $\omega \in \Omega$;

$t_{a}^{\oplus}(\cdot)$ : travel time on link $a, a \in A$ under demand realization $\omega \in \Omega$;

$t_{a}^{k, \omega}(\cdot)$ : the link travel time for the $k$ th, $k \in K$ users under demand realization $\omega \in \Omega$; $\delta_{a, r}^{k, w}$ : link-path incidence; $\delta_{a, r}^{k, w}=1$ if link $a$ is on the travel route $r, r \in R_{w}^{p}$ for the $k$ th, $k \in K$ users' trips of OD pair $w, w \in W$, and 0 otherwise;

$q_{w}^{k, \omega}:$ travel demand associated with $k$ th, $k \in K$ users and OD pair $w, w \in W$ under demand realization $\omega \in \Omega$;

$\operatorname{Pr}^{\Phi}$ : the probability that the demand $\omega \in \Omega$ is realized;

$\rho$ : the weighted parameter for the expected total travel time of auto travelers, $\rho \in[0,1]$;

$B$ : the total budget for the EV network design;

$C_{a}^{0}$ : the unit lane capacity of physical link $a, a \in A$;

$L_{a}^{0}$ : the initial number of lanes of link $a, a \in A$ (before setting EV lanes);

$C_{a}$ : the capacity of link $a, a \in A \cup \widehat{A} \cup \widetilde{A}$;

$t_{a}^{0}$ : free-flow travel time of link $a, a \in A$;

$\alpha_{a}, \beta_{a}$ : two coefficients in the travel time function;

$b_{a}(\cdot)$ : the construction cost function with respect to decision variables $\hat{x}_{a}$ and $\tilde{x}_{a}$ and the parameter $\gamma_{a}$.

To facilitate model formulation, we make three assumptions below.

Assumption 4. Link travel time function $t_{a}^{\Phi}\left(v_{a}^{\Phi}\right)$ is a differentiable strictly increasing function with respect to the aggregate link flow $v_{a}^{\infty}$; that is, $d t_{a}\left(v_{a}\right) / d v_{a}>0$.

Assumption 5. The network design problem takes into account uncertain peak-hour travel demands for both auto and EV users. The random demands for the two classes of users are assumed to follow known probability distributions.

Assumption 6. The EV lanes are assumed to open during the peak-hour period and private cars are not allowed to use any EV lanes. The network planner evaluates the network performance with constantly open EV lanes.

Assumption 6 implies EVs can either make use of EV lanes or move on regular lanes that eventually generate 


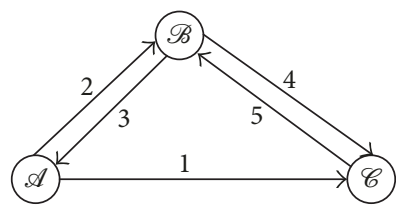

(a) The original network

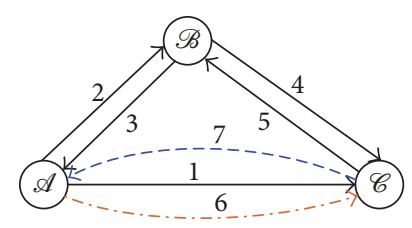

(b) The reconstructed network

Figure 2: An illustrative example to reconstruct the network.

mixed traffic. We only study an extreme network state with constantly open EV lanes. One may argue that some individuals cannot tolerate building exclusive EV lanes to compete against limited road resources, which may lead to a more congested network. This concern could be greatly alleviated under traffic control management. When the traffic control center receives callings of EVs, private cars are not allowed to use EV lanes; otherwise, the EV lanes are fully free for use. Actually, an interesting extension could be made in the future to simultaneously optimize the EV-NDP scheme and working state setting of EV lanes. The working state setting is to determine an optimal active time of EV lanes (i.e., an optimal proportion to the entire peak-hour period).

2.3. Network Reconstruction. Partitioning a regular road generates two independent derivative products, an EV link and a new regular link with reduced capacity (one may have no capacity), and as a result, setting EV lanes changes the network's topological structures for auto and EV users. It is not surprising that the access rights of private cars might be restricted due to the implementation of EV-NDP schemes. On the one hand, private cars cannot use newly added EV links. On the other hand, private cars that pass new regular links with possibly reduced capacities would face more congested traffic and take longer travel time. In turn, EVs enjoy more road infrastructures and more flexible routing choices due to the newly added EV links on the network. In addition, incorporating inverse-direction EV lanes would make vehicle accessibilities more complicated.

To properly address the distinct vehicle accessibilities, we build a unified urban transportation network that can better accommodate two classes of users. In detail, for each candidate link $a, a \in \bar{A}$ in the network, we add two dummy EV links, one normal-direction EV link $\widehat{a}$ and one inversedirection EV link $\tilde{a}$, and map them together as a triplet $\langle a, \widehat{a}, \widetilde{a}\rangle$. As shown in Figure 2, let us build an EV lane on the candidate Link 1 . A corresponding triplet $\langle 1,6,7\rangle$ is thus constructed and $\widehat{A}=\{6\}$ and $\widetilde{A}=\{7\}$. We then can see that auto drivers can use the roads in the subnetwork $G=(N, A)$, where $A=\{1,2,3,4,5\}$, and EVs commute on the network $G=\left(N, A^{\prime}\right)$, where $A^{\prime}=A \cup\{6,7\}$. Note that if an EV lane is set, Link 1 drops one-lane capacity. Evidently, the unified network is general enough to characterize the differentiated vehicle accessibilities of two classes of users.

We next need to identify the association between decision variables and network parameters. We now focus on determining link capacities. Define the EV-NDP scheme as $\mathbf{x}:=(\widehat{\mathbf{x}}, \widetilde{\mathbf{x}})$, where $\widehat{\mathbf{x}}:=\left(\widehat{x}_{a}, a \in \widehat{A}\right)$ and $\widetilde{\mathbf{x}}:=\left(\widetilde{x}_{a}, a \in \widetilde{A}\right)$. Under a given EV-NDP scheme $\mathbf{x}$, the capacities of links contained in every triplet $\langle a, \widehat{a}, \widetilde{a}\rangle$ can be determined by

$$
\begin{aligned}
C_{a} & =C_{a}^{0}\left(L_{a}^{0}-\widehat{x}_{\widehat{a}}-\widetilde{x}_{\tilde{a}}\right), \quad a \in \bar{A}, \\
C_{a^{\prime}} & =C_{a}^{0} \widehat{x}_{\widehat{a}}, \quad \widehat{a} \in \widehat{A} \\
C_{\widetilde{a}} & =C_{a}^{0} \widetilde{x}_{\widetilde{a}}, \quad \widetilde{a} \in \widetilde{A}
\end{aligned}
$$

where $L_{a}^{0}$ is the number of original lanes of physical link $a$ before setting EV lanes. Here, the unit lane capacity is evenly divided according to the lane number and total link capacity. In practice, on each selected road, at most one EV lane (either normal-direction or inverse-direction EV lane) could be built. We thus have the following feasibility constraint:

$$
\widehat{x}_{\widehat{a}}+\tilde{x}_{\tilde{a}} \leq 1, \text { for each triplet }\langle a, \widehat{a}, \widetilde{a}\rangle .
$$

To distinguish the access rights of EV links $a \in \widehat{A} \cup \widetilde{A}$ for two types of users, we need to redefine the link travel cost functions for the two classes of users:

$$
\begin{aligned}
& t_{a}^{e, \oplus}=t_{a}^{\oplus}\left(v_{a}^{\oplus}\right), \quad \text { for EV users, } a \in \widehat{A} \cup \widetilde{A}, \\
& t_{a}^{p, \omega}=t_{a}^{\oplus}\left(v_{a}^{\oplus}\right)+\widehat{x}_{a} \bar{\tau}, \quad \text { for auto users, } a \in \widehat{A}, \\
& t_{a}^{p, \omega}=t_{a}^{\oplus}\left(v_{a}^{\oplus}\right)+\tilde{x}_{a} \bar{\tau}, \quad \text { for auto users, } a \in \widetilde{A},
\end{aligned}
$$

where $t_{a}^{e, \omega}$ and $t_{a}^{p, \omega}$ are, respectively, the redefined link travel cost functions of EV users and auto users under demand realization $\omega \in \Omega ; \bar{\tau}$ is a sufficiently large road access charge to prohibit auto travelers from using EV links. The different access rights on EV links of the two classes of users could be easily verified in (5).

2.4. The EV-NDP Model. In this paper, we aim to investigate an EV-NDP in the strategic planning level, which takes into account long-term uncertainties of auto and EV demands. In spirit of the classical NDP modeling framework, the EVNDP can be formulated as a bilevel programming model. The upper-level problem aims to minimize the weighted sum of the expected total travel time (ETTT) of auto and EV travelers subject to a series of constraints, while the lower-level problem is to characterize the travelers' route 
choice behaviors under given demand realizations. We thus formulate the upper-level problem as follows:

$$
\begin{aligned}
& g(\mathbf{x})=\min _{\mathbf{x}} \rho \sum_{\forall}[\left.\operatorname{Pr}^{\Phi} \sum_{\forall a} v_{a}^{p, \Phi} t_{a}^{p, \omega}\left(v_{a}^{\Phi}, \widehat{x}_{a}, \tilde{x}_{a}\right)\right] \\
&+(1-\rho) \sum_{\forall \omega}\left[\operatorname{Pr}^{\Phi} \sum_{\forall a} v_{a}^{e, \omega} t_{a}^{e, \Phi}\left(v_{a}^{\Phi}, \widehat{x}_{a}, \tilde{x}_{a}\right)\right]
\end{aligned}
$$

s.t. $\quad(4)$

$$
\begin{aligned}
& \sum_{a \in \widehat{A}} b_{a}\left(\widehat{x}_{a}, \gamma_{a}\right)+\sum_{a \in \widetilde{A}} b_{a}\left(\tilde{x}_{a}, \gamma_{a}\right) \leq B, \\
& \widehat{x}_{\widehat{a}}, \tilde{x}_{\tilde{a}} \in\{0,1\}, \forall \widehat{a} \in \widehat{A}, \tilde{a} \in \widetilde{A},
\end{aligned}
$$

where $\rho \in[0,1]$ is the weighted parameter. The auxiliary variable $v_{a}^{\omega}$ is the aggregate link flow given that the demand $\varpi \in \Omega$ is realized, which is further specified as

$$
v_{a}^{\Phi}=v_{a}^{p, \omega}+\eta v_{a}^{e, \omega}, \quad \forall a \in A,
$$

where $\eta$ is the coefficient to convert the $\mathrm{EV}$ traffic volumes to passenger car equivalent (PCU) traffic volumes (empirically, $\eta \geq 1.0$ ).

The traffic flows $v_{a}^{p, \omega}$ and $v_{a}^{e, \omega}$ to the upper level are obtained by solving a biclass user equilibrium in the following lower-level model for each demand realization $\omega \in \Omega$ :

$$
\begin{aligned}
& \min _{\substack{p, \omega \\
v_{a}^{p, Q}, v_{a}^{, \omega}}} \sum_{\forall a} \int_{0}^{v_{a}^{\infty}} t_{a}^{\Phi}\left(w, \widehat{x}_{a}, \tilde{x}_{a}\right) \mathrm{d} w \\
& \text { s.t. } \sum_{r \in R_{w}^{k}} f_{r, w}^{k, \omega}=q_{w}^{k, \omega}, \quad w \in W, k \in K, \forall \omega, \\
& v_{a}^{k, \omega}=\sum_{w \in W} \sum_{r \in R_{w}^{k}} f_{r, w}^{k, \omega} \delta_{a, r}^{k, w}, \\
& a \in A^{\prime}, k \in K, \forall \oplus, \\
& f_{r, w}^{k, \omega} \geq 0, \quad r \in R_{w}^{k}, k \in K, w \in W, \forall \varpi,
\end{aligned}
$$

and the linear constraints (9) of the aggregate link flows.

With the strictly increasing link travel time function (Assumption 4), the biclass user equilibrium model in the lower level is a strictly convex minimization problem. That is, under given EV-NDP scheme $\mathbf{x}$ and demand realization $\omega$, solving the user equilibrium generates a unique traffic flow pattern $\left(v_{a}^{p, \omega}, v_{a}^{e, \omega}\right)$.

As the EV travel demand for each OD pair is far less than auto commuting demand, the magnitude of the ETTT of auto travelers would be significantly greater than that of EV users (see the third case of 68,181.82 versus 1,740.55 in the illustrative example). The huge difference in magnitude leads to difficulty in analyzing the impact of the weighted parameter setting in designing desirable EV-NDP schemes. To better examine the influence of the weighted parameter on balancing the two network performance measures, we introduce two performance benchmarks and redefine a normalized design objective function. Let $g^{p, 0}$ and $g^{e, 0}$, respectively, represent the ETTTs of auto and EV travelers without implementing any EV-NDP schemes, which can be easily obtained by solving the lower-level user equilibrium model (10). Based on the two performance benchmarks $g^{p, 0}$ and $g^{e, 0}$, we thus can rewrite the normalized design objective function $g(\mathbf{x})$ as

$$
\begin{aligned}
\min _{\mathbf{x}} & \left\{\frac{\rho}{g^{p, 0}} \sum_{\forall \varpi} \operatorname{Pr}^{\Phi} \sum_{\forall a} v_{a}^{p, \omega} t_{a}^{p, \omega}\left(v_{a}^{\Phi}, \widehat{x}_{a}, \tilde{x}_{a}\right)\right. \\
+ & \left.\frac{1-\rho}{g^{e, 0}} \sum_{\forall \omega} \operatorname{Pr}^{\Phi} \sum_{\forall a} v_{a}^{e, \omega} t_{a}^{e, \Phi}\left(v_{a}^{\Phi}, \widehat{x}_{a}, \tilde{x}_{a}\right)\right\} .
\end{aligned}
$$

Therefore, the EV-NDP model can be expressed by (11) and is subject to the feasible solution space defined by constraints (4) and (7)-(10).

We further give a brief remark on designing the two aforementioned EV-NDP schemes: the scheme with pure normal-direction EV lanes and the scheme with flexible inverse-direction EV lanes. It is not difficult to find that the former scheme is a special case of the latter one. Setting all decision variables $\widetilde{\mathbf{x}}=\mathbf{0}$ of an inverse-direction EV-NDP scheme generates a pure normal-direction EV-NDP scheme.

\section{Solution Algorithms}

As the proposed bilevel programming model is nonlinear and nonconvex in nature, it would be rather difficult to solve the EV-NDP model using conventional solution methods. Moreover, the incorporation of demand uncertainties results in a higher computational complexity, which makes the model more intractable to be solved. At early stages, a few greedy/descent search algorithms were developed to solve the NDPs with deterministic demands, including the Hooke-Jeeves algorithm [12], sequential quadratic programming method [38], augmented Lagrangian algorithm [39], and gradient/conjugate gradient/quasi-Newton projection methods [40]. In the last decade, more and more researchers have paid attention to global optimization methods, including mixed-integer linear programming (MILP) method [3335], benders decomposition algorithm [41], and system optimum-relaxation based method [36].

However, the above-mentioned solution algorithms are inapplicable for solving the EV-NDP model. On the one hand, we can hardly use descent search algorithms to solve the EVNDP model due to two reasons: first, the search space is large and highly complex; second, it is intractable to obtain effective descent gradients for a discrete bilevel optimization model, especially in the presence of stochastic demands. On the other hand, incorporating demand randomness requires us to address a large number of demand realizations and consequently makes it almost impossible to design a global optimization method.

Fortunately, metaheuristic solution algorithms can provide us with alternative options. Previous studies clearly indicated that the genetic algorithm (GA) is one of the most prevailing and recommendable tools to solve NDP models $[18,42]$. For example, Chen and Yang [23] proposed a simulation-based GA procedure to solve expected-value 


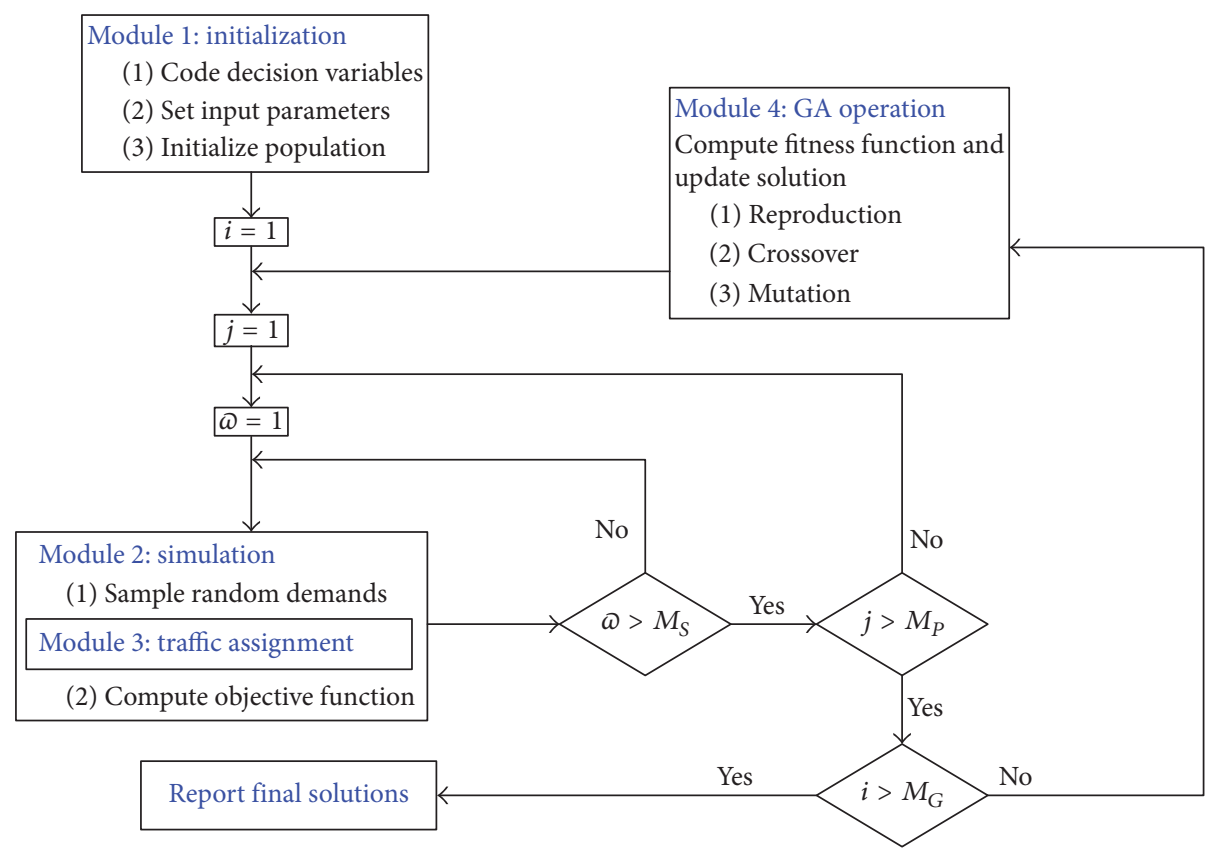

FIgURE 3: The simulation-based genetic algorithm for the EV-NDP model.

and chance-constraint NDP models that considered both spatial equity and demand uncertainty. Chootinan et al. [24] presented a bilevel capacity reliability-based network design model, where the upper-level model was solved by MonteCarlo simulation-based GA and probit-based stochastic user equilibrium in the lower level was solved by the method of successive averages. Chen et al. [20] and Chen and Xu [19] applied the GA to solve multiobjective NDPs with demand uncertainties. Ukkusuri et al. [8] developed a GA to solve a robust NDP, in which the design objective was to minimize the weighted sum of the expected total travel time and related standard variance. Sharma et al. [21, 22] applied the GA to solve similar mean-variance form robust NDPs with random demands. More information about the GA in solving stochastic NDPs can be found in Chen et al. [18].

In this paper, we also design a simulation-based GA to solve the proposed EV-NDP model. Figure 3 presents the explicit framework of the proposed GA. It works with four submodules: initialization module, simulation module, traffic assignment module, and GA operation module. Each of them is elaborated below:

(1) Initialization module: this module translates the decision variables to GA codes, sets input parameters of experiments, and randomly generates an initial population.

(2) Simulation module: it is used to produce a series of demand realizations according to given demand probability distributions. Next, combined with traffic patterns obtained by solving the lower-level user equilibrium under each demand realization, we use Monte-Carlo simulation to estimate the design objective function using sample average approximation (SAA). Interested readers can refer to Sharma et al. [22] for other attractive sampling approximation techniques, for example, randomized quasi-MonteCarlo sampling and single-point approximation.

(3) Traffic assignment module: it characterizes the users' route choice behaviors associated with the given demand realization and EV-NDP scheme. The widely applied Frank-Wolfe algorithm is used to solve the lower-level traffic assignment problem.

(4) GA operation module: this module aims to generate the next trial solution via various genetic operations. Applying a series of GA operations, including reproduction, crossover, and mutation, produces better offspring. More details could be found in Ukkusuri et al. [8].

Algorithm 1 presents the detailed procedure to solve the developed EV-NDP model.

In solving the EV-NDP model, we take an improved strategy of population initialization to filter out infeasible populations that violate the feasibility constraint (4) and budget constraint (7). Furthermore, a population updating strategy (e.g., reinitializing population frequently) can be used to avoid a local optimum as far as possible.

\section{Numerical Examples}

In this section, numerical examples are provided to evaluate the developed EV-NDP model and the computation efficiency of the proposed algorithm. The programming code is compiled by $\mathrm{VC}++$ and run on Windows 7 system with the following attributes: Intel Core i5-2400 $3.1 \mathrm{GHz} \times 2$ and $4 \mathrm{~GB}$ RAM.

As shown in Figure 4, a four-node urban transportation network with nine links is used for the numerical experiments. Link parameter settings including the number 


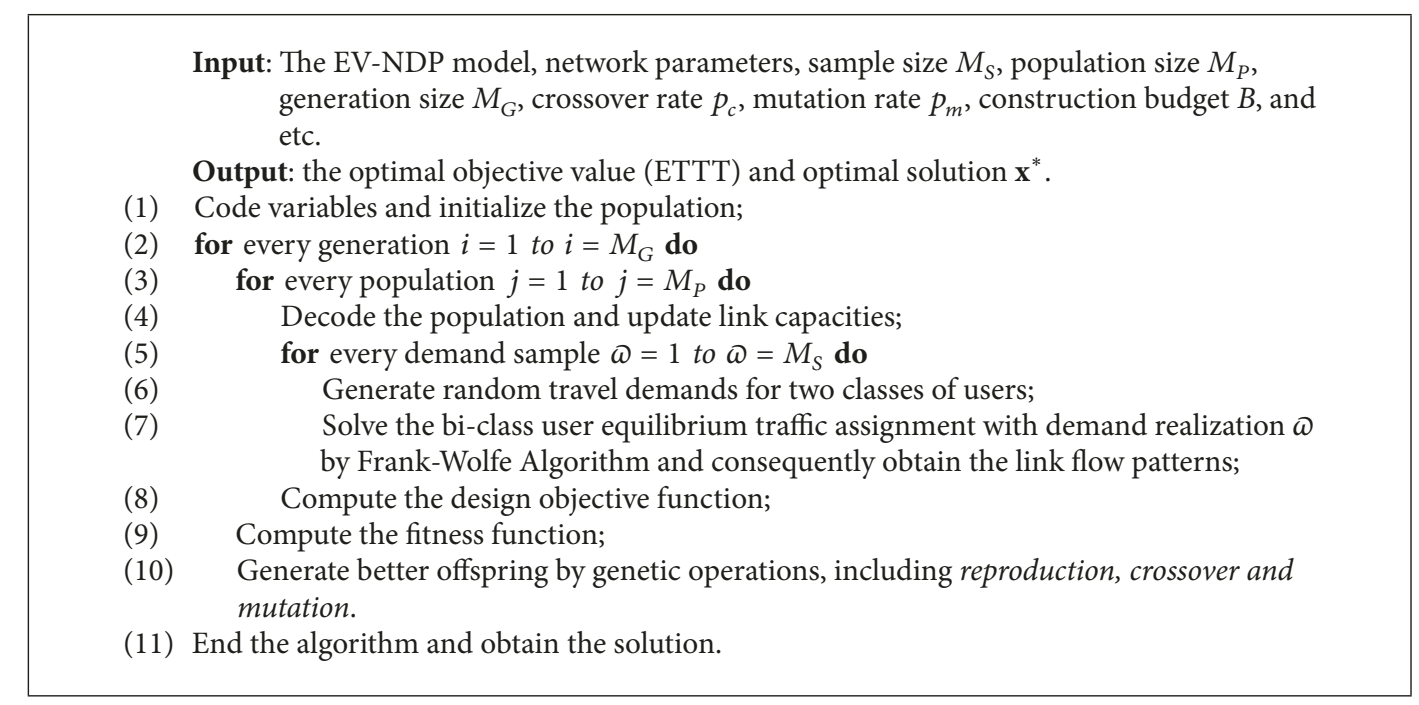

Algorithm 1: Simulation-based genetic algorithm.

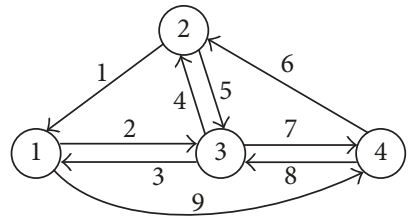

FIGURE 4: The used transportation network.

of lanes, unit lane capacity, and free-flow travel time are provided in Table 2.

Six OD pairs are taken into account. For each OD pair, the auto and EV travel demands are all assumed to follow given normal distributions. Detailed parameters of respective demand distributions are given in Table 3. below:

The link travel time function (BPR function) is given

$$
t_{a}^{\varrho}\left(v_{a}^{\varrho}\right)=t_{a}^{0}\left(1+0.15\left(\frac{v_{a}^{\varrho}}{C_{a}}\right)^{4}\right),
$$

where $v_{a}^{\oplus}=v_{a}^{p, \omega}+1.5 v_{a}^{e, \omega}$

A linear function is used to determine the construction cost of setting an EV lane:

$$
b_{a}= \begin{cases}\left(10.0+10.0 t_{a}^{0}\right) \widehat{x}_{a}, & a \in \widehat{A}, \\ \left(10.0+10.0 t_{a}^{0}\right) \widetilde{x}_{a}, & a \in \widetilde{A} .\end{cases}
$$

Other parameters used in the GA procedure are listed in Table 4.

For both normal-direction and inverse-direction EVNDP schemes, we have tested the experiments by changing the weighted parameter in the design objective function (an equal increment of 0.1 is set). In addition to applying the simulation-based GA, we solve the normal-direction EV-NDP schemes by the enumeration method in order to compare the solution quality and computational efficiency.
Solving the lower-level traffic assignment problem of the normal-direction EV-NDP generates the benchmark measures where $g^{p, 0}=113,779.42$ and $g^{e, 0}=3,395.93$ (note that solving the user equilibrium model of the inverse-direction EV-NDP yields $g^{p, 0}=113,777.49$ and $g^{e, 0}=3,395.63$; the small differences are partly due to computational accuracy and partly resulted from two different feasible path sets between the normal-direction and inverse-direction networks).

4.1. Computational Results. Table 5 shows the outcomes (including the optimal solution-the "optimal solution" obtained by the simulation-based GA may not be globaloptimal but only local-optimal-ETTTs for two classes of users, the objective value, and computational time) of the normal-direction EV-NDP schemes. Table 6 provides the computed results of the inverse-direction EV-NDP schemes. As shown in Table 5, for the normal-direction EV-NDPs, the simulation-based GA and enumeration method generate identical results except for the case with $\rho=0.1$. In what follows, we make further discussions on the computed results and expect to gain some insightful findings.

4.2. The Value of Incorporating Inverse-Direction EV Lanes. We first analyze the importance of incorporating inversedirection EV lanes. The objective values of the normaldirection and inverse-direction EV-NDPs with different weighted parameter settings are illustrated in Figure 5 for an easy comparison. It is not surprising to realize that, with a given weighted parameter, the scheme considering inversedirection EV lanes performs better than the corresponding normal-direction EV-NDP in terms of the design objective value since the latter scheme is a special case of the inversedirection EV-NDP. Take the experiment with $\rho=0.2$ as an example. We obtain a global-optimal objective value $g^{*}=$ 99.215\% by solving the normal-direction EV-NDP scheme with the enumeration method. Solving the inverse-direction EV-NDP scheme by the simulation-based GA generates an 
TABLE 2: The detailed link parameter setting.

\begin{tabular}{lccccccccc}
\hline Link number & 1 & 2 & 3 & 4 & 5 & 6 & 7 & 8 & 9 \\
\hline$C_{a}^{0}$ & 1200 & 1000 & 1000 & 800 & 800 & 1200 & 1000 & 1000 \\
$L_{a}^{0}$ & 3 & 2 & 2 & 2 & 2 & 3 & 2 & 2 \\
$t_{a}^{0}$ & 10 & 8 & 8 & 4 & 4 & 6 & 5 & 5 \\
\hline
\end{tabular}

TABLE 3: Travel demand distributions for two classes of users.

\begin{tabular}{lcccccc}
\hline OD pair & $1 \rightarrow 2$ & $1 \rightarrow 4$ & $2 \rightarrow 1$ & $2 \rightarrow 4$ & $4 \rightarrow 1$ & $4 \rightarrow 2$ \\
\hline Demand for cars & $\left(1000,100^{2}\right)$ & $\left(2000,100^{2}\right)$ & $\left(1000,100^{2}\right)$ & $\left(2200,100^{2}\right)$ & $\left(1800,100^{2}\right)$ & $\left(2100,100^{2}\right)$ \\
Demand for EVs & $\left(50,5^{2}\right)$ & $\left(50,5^{2}\right)$ & $\left(50,5^{2}\right)$ & $\left(50,5^{2}\right)$ & $\left(50,5^{2}\right)$ & $\left(50,5^{2}\right)$ \\
\hline
\end{tabular}

Note. Numerics $\left(a, b^{2}\right)$ are the mean and variance of a given normal distribution for each OD pair.

TABLE 4: Other parameters used in the numerical experiments.

\begin{tabular}{|c|c|}
\hline \multirow[b]{2}{*}{ Population size } & $\begin{array}{c}M_{P}=10 \text { for the normal-direction } \\
\text { EV-NDP scheme }\end{array}$ \\
\hline & $\begin{array}{c}M_{P}=30 \text { for the inverse-direction } \\
\text { EV-NDP scheme }\end{array}$ \\
\hline \multirow{2}{*}{ Generation size } & $\begin{array}{c}M_{G}=50 \text { for the normal-direction } \\
\text { EV-NDP scheme }\end{array}$ \\
\hline & $\begin{array}{c}M_{G}=80 \text { for the inverse-direction } \\
\text { EV-NDP scheme }\end{array}$ \\
\hline Probability of crossover & 0.75 \\
\hline Total investment budget & $B=400$ \\
\hline Probability of mutation & 0.1 \\
\hline Sample size & $M_{S}=500$ \\
\hline
\end{tabular}

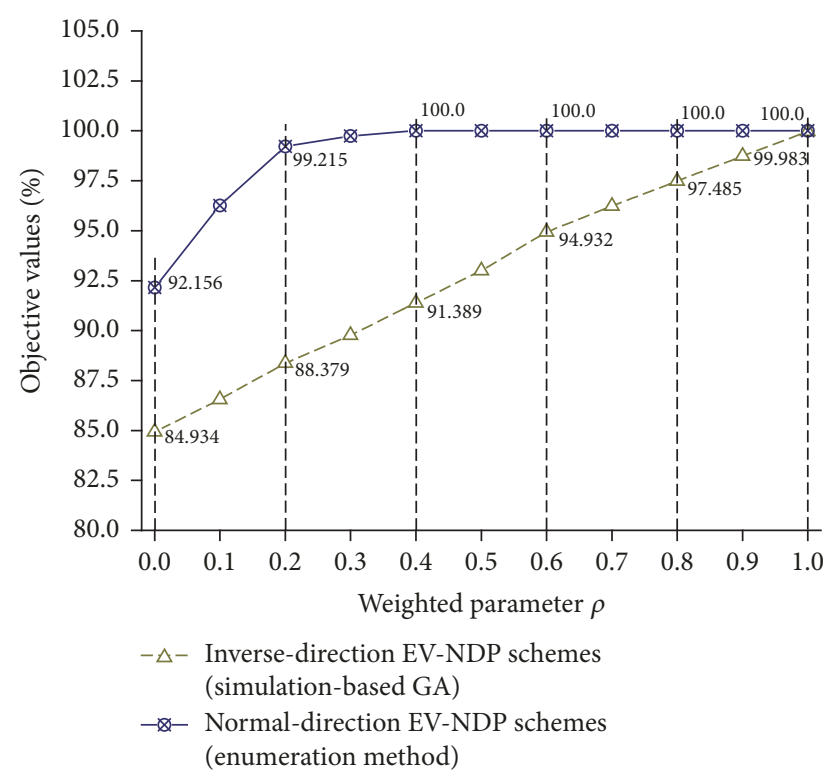

FIGURE 5: Comparison between the normal-direction and inversedirection EV-NDPs.

optimal objective value $g^{*}=88.379 \%$. Although the solution of the inverse-direction EV-NDP scheme leads to a local optimum (the solution $\left(\widehat{\mathbf{x}}^{*}=\mathbf{0}, \widetilde{\mathbf{x}}^{*}=(1,0,0,0,0,1,0,0,1)\right)$ yields a better objective value of $g^{*}=88.162 \%$ ), it still remarkably improves the travel efficiencies of both classes of users. It can be seen from Tables 5 and 6 that both classes of users benefit from incorporating inverse-direction EV lanes: the ETTT of private cars drops from 119,101.05 to 116,230.05 and that of EVs decreases from 3,322.89 to 2,884.32. In summary, incorporating inverse-direction EV lanes in the EV-NDP offers a chance to design more attractive EV-NDP schemes.

4.3. The Impact of Weighted Parameter Setting. As shown in Figure 5, for both EV-NDP schemes, the design objective values climb steadily with an increasing weighted factor. As stated in the Introduction, the nature of EV-NDP is to reallocate the road capacity to each class of travelers and the total capacity on each link is fixed. Consequently, allocating part of the road capacity to EVs often makes private cars suffer a more congested traffic condition unless a paradox occurs (this indeed happens and will be discussed later). In this sense, balancing two conflicting objectives of minimizing ETTTs of private cars and EVs eventually creates, to a certain extent, a zero-sum game. As shown in Table 5, the ETTT for EVs falls monotonically when a higher priority is given to enhance EVs' travel efficiency. On the contrary, the ETTT for private cars rises as the weighted parameter increases. A similar trend can be found in the outcomes of inverse-direction EV-NDP schemes, although it is not strictly monotone due to local-optimal solutions attained by the simulation-based GA. Overall, the developed weighted-sum EV-NDP model is capable of finding an appropriate tradeoff between two conflicting design objectives for two classes of users.

4.4. Solution Convergence, Quality, and Computational Efficiency. We now turn to analyze the solution convergence, quality, and computational efficiency of the proposed algorithm. Figure 6 clearly displays the convergence of the proposed simulation-based GA in solving both the normaldirection and the inverse-direction EV-NDPs. With no doubt, the simulation-based GA is able to obtain localoptimal solutions.

Let us next examine the solution quality. The results of normal-direction EV-NDPs solved by the simulation-based GA and enumeration method are depicted in Figure 7 for a straightforward comparison. It can be observed that the outcomes from the simulation-based GA are identical to 
TABLE 5: The outcomes for the normal-direction EV-NDP schemes.

\begin{tabular}{lccccc}
\hline Weighting factor $\rho$ & Optimal solution $\widehat{\mathbf{x}}^{*}$ & ETTT for cars & ETTT for EVs & Objective value $g^{*}\left(\widehat{\mathbf{x}}^{*}\right)(\%)$ & Computational time $(\mathrm{s})$ \\
\hline 0.00 & $(0,0,1,0,1,1,1,0,1)$ & $151,763.02$ & $3,129.54$ & 92.156 & $1,452.29$ \\
0.10 & $(0,0,1,0,1,0,1,1,1)$ & $148,924.72$ & $3,140.92$ & 96.331 & $1,471.84$ \\
& $\underline{(0,0,1,1,1,1,1,1,0)}$ & $\underline{146,643.41}$ & $\underline{3,146.02}$ & $\underline{96.265}$ & $\frac{1,579.12}{99.215}$ \\
0.20 & $(0,0,1,0,0,0,0,1,1)$ & $119,101.05$ & $3,322.89$ & 99.748 & $1,522.38$ \\
0.30 & $(0,0,1,0,0,0,0,1,0)$ & $117,732.08$ & $3,333.16$ & 100.00 & $1,526.89$ \\
0.40 & $(0,0,0,0,0,0,0,0,0)$ & $113,779.42$ & $3,395.93$ & 100.00 & $1,467.46$ \\
0.50 & $(0,0,0,0,0,0,0,0,0)$ & $113,779.42$ & $3,395.93$ & 100.00 & $1,481.86$ \\
0.60 & $(0,0,0,0,0,0,0,0,0)$ & $113,779.42$ & $3,395.93$ & 100.00 & $1,567.94$ \\
0.70 & $(0,0,0,0,0,0,0,0,0)$ & $113,779.42$ & $3,395.93$ & 100.00 & $1,542.40$ \\
0.80 & $(0,0,0,0,0,0,0,0,0)$ & $113,779.42$ & $3,395.93$ & 100.00 & $1,521.61$ \\
0.90 & $(0,0,0,0,0,0,0,0,0)$ & $113,779.42$ & $3,395.93$ & 100.00 & $1,508.46$ \\
1.00 & $(0,0,0,0,0,0,0,0,0)$ & $113,779.42$ & $3,395.93$ & $1,529.05$ \\
\hline
\end{tabular}

Note. For the experiment with $\rho=0.1$, the computed results solved by the enumeration method are underlined, while the results obtained by the simulationbased GA are presented without underline.

TABLE 6: The outcomes for the inverse-direction EV-NDP schemes.

\begin{tabular}{|c|c|c|c|c|c|}
\hline Weighting factor $\rho$ & $\rho$ Optimal solution $\mathbf{x}^{*}=\left(\widehat{\mathbf{x}}^{*}, \widetilde{\mathbf{x}}^{*}\right)$ & ETTT for cars & ETTT for EVs & Objective value $g^{*}\left(\widehat{\mathbf{x}}^{*}\right)(\%)$ & Computational time (s) \\
\hline 0.00 & $\widehat{\mathbf{x}}^{*}=\mathbf{0}, \widetilde{\mathbf{x}}^{*}=(1,0,0,1,0,1,0,0,1)$ & $116,502.41$ & $2,884.32$ & 84.935 & $25,512.50$ \\
\hline 0.10 & $\widehat{\mathbf{x}}^{*}=\mathbf{0}, \widetilde{\mathbf{x}}^{*}=(1,0,0,0,0,1,0,0,1)$ & $114,996.07$ & $2,884.32$ & 86.548 & $25,836.17$ \\
\hline 0.20 & $\widehat{\mathbf{x}}^{*}=\mathbf{0}, \widetilde{\mathbf{x}}^{*}=(1,1,0,0,0,1,0,0,1)$ & $116,230.05$ & $2,884.32$ & 88.379 & $26,180.31$ \\
\hline 0.30 & $\widehat{\mathbf{x}}^{*}=\mathbf{0}, \widetilde{\mathbf{x}}^{*}=(1,0,0,0,0,1,0,0,1)$ & $114,996.07$ & $2,884.32$ & 89.775 & $25,314.65$ \\
\hline 0.40 & $\widehat{\mathbf{x}}^{*}=\mathbf{0}, \widetilde{\mathbf{x}}^{*}=(1,0,0,0,0,1,0,0,1)$ & $114,996.07$ & $2,884.32$ & 91.389 & $25,788.18$ \\
\hline 0.50 & $\widehat{\mathbf{x}}^{*}=\mathbf{0}, \widetilde{\mathbf{x}}^{*}=(1,0,0,0,0,1,0,0,1)$ & $114,996.07$ & $2,884.32$ & 93.002 & $25,779.22$ \\
\hline 0.60 & $\widehat{\mathbf{x}}^{*}=\mathbf{0}, \widetilde{\mathbf{x}}^{*}=(1,1,0,0,0,1,0,0,0)$ & $114,740.04$ & $2,922.68$ & 94.932 & $25,747.76$ \\
\hline 0.70 & $\widehat{\mathbf{x}}^{*}=\mathbf{0}, \widetilde{\mathbf{x}}^{*}=(1,0,0,0,0,1,0,0,1)$ & $114,996.07$ & $2,884.32$ & 96.229 & $25,739.35$ \\
\hline 0.80 & $\widehat{\mathbf{x}}^{*}=\mathbf{0}, \widetilde{\mathbf{x}}^{*}=(1,0,0,0,0,1,0,0,0)$ & $113,764.05$ & $2,970.79$ & 97.485 & $25,749.86$ \\
\hline 0.90 & $\widehat{\mathbf{x}}^{*}=\mathbf{0}, \widetilde{\mathbf{x}}^{*}=(1,0,0,0,0,1,0,0,0)$ & $113,764.05$ & $2,970.79$ & 98.736 & $26,324.82$ \\
\hline 1.00 & $\widehat{\mathbf{x}}^{*}=\mathbf{0}, \widetilde{\mathbf{x}}^{*}=(1,0,0,0,0,0,0,0,0)$ & $113,759.80$ & $3,286.19$ & 99.983 & $25,750.46$ \\
\hline
\end{tabular}

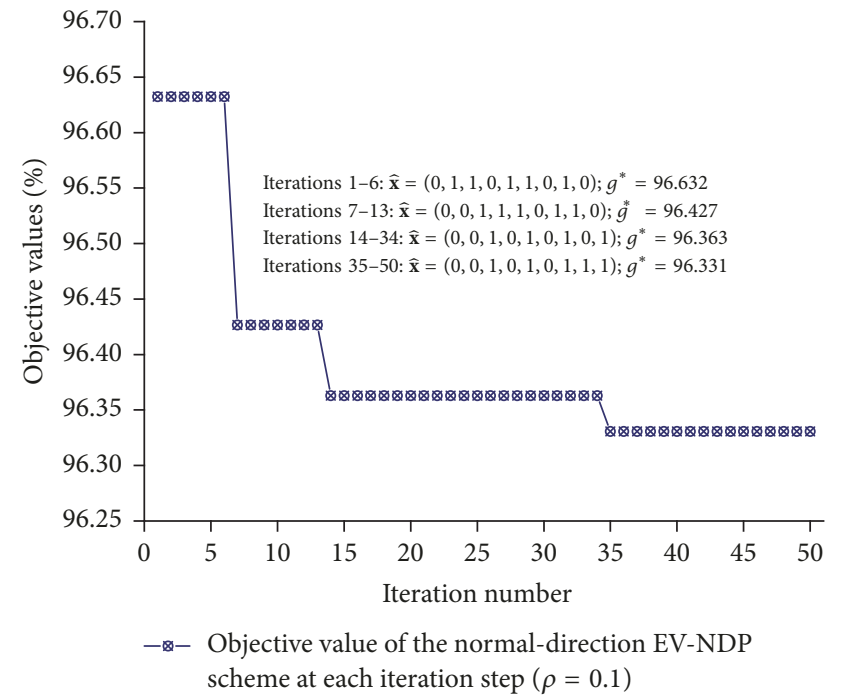

(a) Normal-direction EV-NDP with $\rho=0.1$

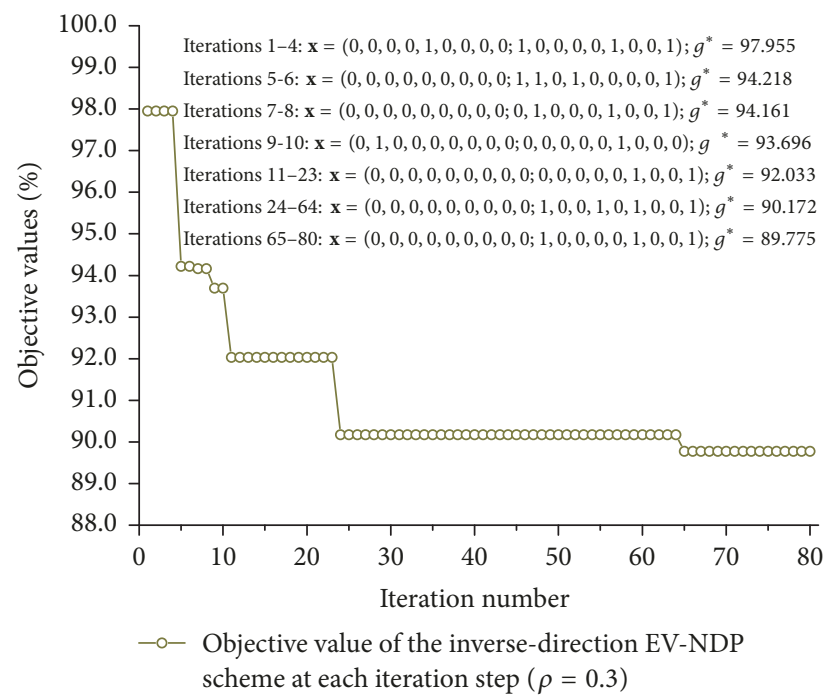

(b) Inverse-direction EV-NDP with $\rho=0.3$

FIgURE 6: Examples to illustrate the convergence of the simulation-based GA. 


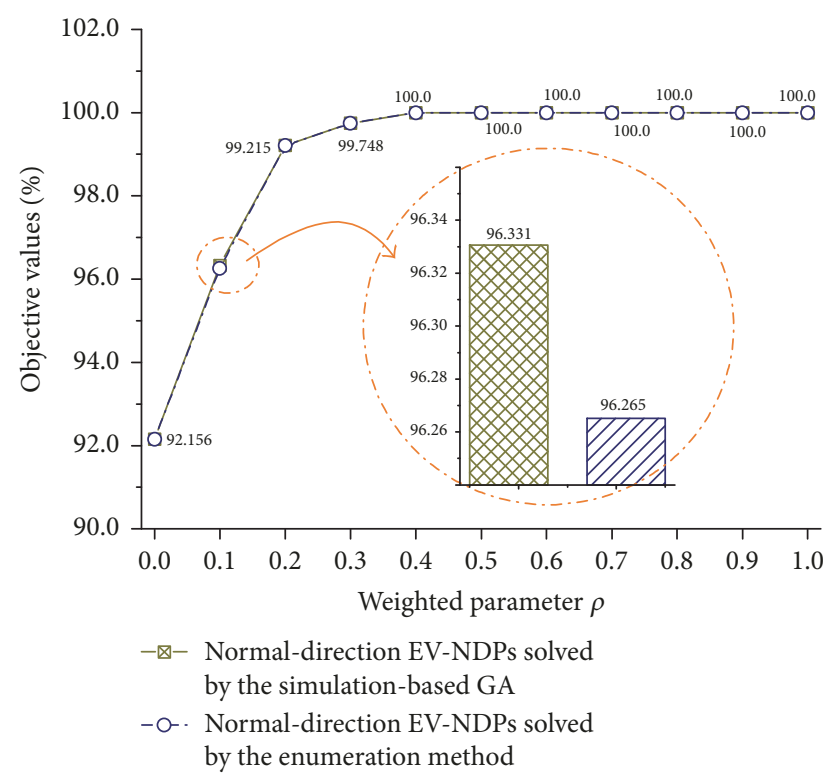

FIGURE 7: Comparison of the results of normal-direction EV-NDPs solved by the simulation-based GA and enumeration method.

those from the enumeration method, except for the case with $\rho=0.1$. Actually, for the experiment with $\rho=0.1$, the optimal objective values solved by the two methods are very close (96.331 versus 96.265). In general, the simulation-based GA can generate satisfactory solutions, although the tested experiments might be network-specific.

We then focus on the computational efficiency of the proposed algorithm. It can be seen in Table 5 that it takes about 25 minutes to solve the normal-direction EV-NDP model by the simulation-based GA $(500=10 \times 50$ solutions are examined). The enumeration method consumes a bit longer computation time since all $512=2^{9}$ combinatorial solutions are computed and compared. A heavy computational burden results from repeatedly solving the lower-level traffic assignment problem until all demand scenarios are realized. The larger the sample size is, the heavier the computational burden is. For each given solution $\mathbf{x}$, it approximately takes $10 \mathrm{sec}-$ onds to calculate the objective value for the inverse-direction EV-NDP model. Recall that more than $262,144=2^{18}$ solutions need to be evaluated. Although the number of feasible solutions could be reduced according to the feasibility constraints (4) and (7), the enumeration searching space is still unbelievably large. It is expected to take more than hundreds of hours to solve the inverse-direction EV-NDP model by the enumeration method. Therefore, it is almost intractable to do that even for a small-scale network. Fortunately, the simulation-based GA offers an alternative to find satisfactory solutions for the inverse-direction EV-NDPs within 7.5 hours.

4.5. An Insightful Finding of the EV-NDP. Last but not least, we obtain an interesting insight into the inverse-direction EVNDP with $\rho=1.0$. Setting the weighted parameter $\rho$ equal to 1.0 implies that the network planner is only concerned about the ETTT of private cars. To achieve such objective, it is always believed that the decision-maker needs to provide infrastructure resources to private cars as much as possible. Usually, building EV lanes diverts the resources away and would make auto users suffer a loss. However, a paradox occurs. As shown in Table 6, the optimal solution suggests constructing an inverse-direction EV lane on Link 1. As a result, both EVs and private cars benefit from this decision, where the ETTT for EVs decreases from 3,395.63 to 3,286.19 and that for private cars goes down slightly from 113,777.49 to $113,759.80$. An inherent reason is that adding an inversedirection EV lane makes Link 1 become more congested, and thus some auto users (OD pair $2 \rightarrow 1$ ) choose to switch to other travel paths/links. This consequently drives the user equilibrium approaching system optimum. It is not difficult to verify that the EV travelers of OD pair $1 \rightarrow 2$ benefit significantly from the new added EV lane.

The lesson we have learned here is that the objectives to maximize the travel efficiencies of both classes of users are not always conflicting but could be reconcilable in some cases. Meanwhile, the occurrence of the above paradox has highlighted the value of incorporating inverse-direction EV lanes once again (such a win-win case does not happen in normal-direction EV-NDPs).

\section{Concluding Remarks}

In this paper, we studied a new network planning problem to build exclusive EV lanes in an urban transportation network, which falls into the category of discrete NDPs. The investigated EV-NDP differs from the classical NDPs in several aspects: it involves two classes of network users and has a distinct network reconstruction principle; auto and EV travelers have differentiated road access rights on the network. To make more tangible and practical planning decisions, random travel demands that follow known probability distributions are taken into account in the EV-NDP. Moreover, inverse-direction EV lanes are incorporated to promote flexible and attractive EV-NDP schemes.

A unified network framework is introduced to properly describe the differentiated road access rights of private cars and EVs. We then formulate a bilevel programming EVNDP model. In the upper level, the network planner intends to minimize the weighted sum of the ETTTs of auto and EV travelers by setting either normal-direction or inversedirection EV lanes on selected roads. In the lower level, a biclass user equilibrium model is used to characterize travelers' route choice behaviors under given demand realizations. To handle the stochasticity of travel demands, a simulationbased GA is proposed to solve the developed EV-NDP model. We further evaluate the developed EV-NDP model and the proposed solution algorithm through numerical examples.

We arrive at a bunch of useful and interesting observations: incorporating inverse-direction EV lanes could help to design more favorable EV-NDP schemes; the simulationbased GA is capable of finding satisfactory solutions and guaranteeing a high solution quality with an acceptable computational burden; an interesting paradox is found where building EV lanes might contribute to reduced traffic congestion for both auto and EV travelers. This observation tells us 
that the intuitively conflicting objectives of the two classes of users could be reconcilable in some cases.

This research makes an effort to fill in the gap in the literature by developing a bilevel optimization model for the EV-NDP. We can further make some interesting extensions. Firstly, a joint optimization of the EV-NDP scheme and working state setting of EV lanes can be considered so that more reasonable planning decisions could be obtained. Secondly, we analyze a static analytical framework of the EVNDP in this paper. Extension to a dynamic environment is a more appealing research direction.

\section{Conflicts of Interest}

The authors declare that there are no conflicts of interest regarding the publication of this paper.

\section{Acknowledgments}

This study has been substantially supported by research grants from the National Natural Science Foundation of China (71601142, 71531011, and 71471134) and Shanghai Pujiang Program (16PJC090).

\section{References}

[1] X. Qu, Y. Yang, Z. Liu, S. Jin, and J. Weng, "Potential crash risks of expressway on-ramps and off-ramps: a case study in Beijing, China," Safety Science, vol. 70, pp. 58-62, 2014.

[2] Y. Kuang, X. Qu, and S. Wang, "A tree-structured crash surrogate measure for freeways," Accident Analysis \& Prevention, vol. 77, pp. 137-148, 2015.

[3] S. K. So and C. F. Daganzo, "Managing evacuation routes," Transportation Research Part B: Methodological, vol. 44, no. 4, pp. 514-520, 2010.

[4] C. F. Daganzo and S. K. So, "Managing evacuation networks," Transportation Research Part B: Methodological, vol. 45, no. 9, pp. 1424-1432, 2011.

[5] Y. Liu, G.-L. Chang, Y. Liu, and X. Lai, "Corridor-based emergency evacuation system for Washington, D.C. system development and case study," Transportation Research Record, no. 2041, pp. 58-67, 2008.

[6] X. Zhang and G.-L. Chang, "The multi-modal evacuation system (MES) for Baltimore metropolitan region," in Proceedings of the 2012 15th International IEEE Conference on Intelligent Transportation Systems, ITSC 2012, pp. 1250-1257, IEEE, Anchorage, AK, USA, September 2012.

[7] Transportation Research Board, "The role of transit in emergency evacuation," Tech. Rep., 2008.

[8] S. V. Ukkusuri, T. V. Mathew, and S. T. Waller, "Robust transportation network design under demand uncertainty," Computer-Aided Civil and Infrastructure Engineering, vol. 22, no. 1, pp. 6-18, 2007.

[9] X. Qu, J. Zhang, and S. Wang, "On the stochastic fundamental diagram for freeway traffic: Model development, analytical properties, validation, and extensive applications," Transportation Research Part B: Methodological, vol. 104, pp. 256-271, 2017.

[10] S. Wang and X. Qu, "Station choice for Australian commuter rail lines: equilibrium and optimal fare design," European Journal of Operational Research, vol. 258, no. 1, pp. 144-154, 2017.
[11] L. J. Leblanc, "An algorithm for the discrete network design problem," Transportation Science, vol. 9, no. 3, pp. 183-199, 1975.

[12] M. Abdulaal and L. J. LeBlanc, "Continuous equilibrium network design models," Transportation Research Part B: Methodological, vol. 13, no. 1, pp. 19-32, 1979.

[13] T. L. Magnanti and R. T. Wong, "Network design and transportation planning: models and algorithms," Transportation Science, vol. 18, no. 1, pp. 1-55, 1984.

[14] H. Yang and M. G. H. Bell, "Models and algorithms for road network design: a review and some new developments," Transport Reviews, vol. 18, no. 3, pp. 257-278, 1998.

[15] Q. Meng and H. Wang, The Transportation Network Design Problems: Recent Advances, Working Paper, Department of Civil and Environmental Engineering, National University of Singapore, 2015.

[16] S. T. Waller and A. K. Ziliaskopoulos, "Stochastic dynamic network design problem," Transportation Research Record, no. 1771, pp. 106-113, 2001.

[17] G. R. Patil and S. V. Ukkusuri, "System-optimal stochastic transportation network design," Transportation Research Record, no. 2029, pp. 80-86, 2007.

[18] A. Chen, Z. Zhou, P. Chootinan, S. Ryu, C. Yang, and S. C. Wong, "Transport network design problem under uncertainty: a review and new developments," Transport Reviews, vol. 31, no. 6, pp. 743-768, 2011.

[19] A. Chen and X. Xu, "Goal programming approach to solving network design problem with multiple objectives and demand uncertainty," Expert Systems with Applications, vol. 39, no. 4, pp. 4160-4170, 2012.

[20] A. Chen, J. Kim, S. Lee, and Y. Kim, "Stochastic multi-objective models for network design problem," Expert Systems with Applications, vol. 37, no. 2, pp. 1608-1619, 2010.

[21] S. Sharma, S. V. Ukkusuri, and T. V. Mathew, "Pareto optimal multiobjective optimization for robust transportation network design problem," Transportation Research Record, no. 2090, pp. 95-104, 2009.

[22] S. Sharma, T. V. Mathew, and S. V. Ukkusuri, "Approximation techniques for transportation network design problem under demand uncertainty," Journal of Computing in Civil Engineering, vol. 25, no. 4, pp. 316-329, 2011.

[23] A. Chen and C. Yang, "Stochastic transportation network design problem with spatial equity constraint," Transportation Research Record, no. 1882, pp. 97-104, 2004.

[24] P. Chootinan, S. C. Wong, and A. Chen, "A reliability-based network design problem," Journal of Advanced Transportation, vol. 39, no. 3, pp. 247-270, 2005.

[25] A. Sumalee, P. Luathep, W. H. K. Lam, and R. D. Connors, "Evaluation and design of transport network capacity under demand uncertainty," Transportation Research Record, no. 2090, pp. 17-28, 2009.

[26] H. Wang, W. H. K. Lam, X. Zhang, and H. Shao, "Sustainable Transportation Network Design with Stochastic Demands and Chance Constraints," International Journal of Sustainable Transportation, vol. 9, no. 2, pp. 126-144, 2015.

[27] A. Karoonsoontawong and S. T. Waller, "Robust dynamic continuous network design problem," Transportation Research Record, no. 2029, pp. 58-71, 2007.

[28] H. K. Lo and W. Szeto, "Time-dependent transport network design: a study of budget sensitivity," Journal of the Eastern Asia Society for Transportation Studies, vol. 5, pp. 1124-1139, 2003. 
[29] S. V. Ukkusuri and G. Patil, "Multi-period transportation network design under demand uncertainty," Transportation Research Part B: Methodological, vol. 43, no. 6, pp. 625-642, 2009.

[30] Q. Meng and H. Yang, "Benefit distribution and equity in road network design," Transportation Research Part B: Methodological, vol. 36, no. 1, pp. 19-35, 2002.

[31] E. Miandoabchi, F. Daneshzand, W. Y. Szeto, and R. Zanjirani Farahani, "Multi-objective discrete urban road network design," Computers \& Operations Research, vol. 40, no. 10, pp. 24292449, 2013.

[32] R. Z. Farahani, E. Miandoabchi, W. . Szeto, and H. Rashidi, "A review of urban transportation network design problems," European Journal of Operational Research, vol. 229, no. 2, pp. 281-302, 2013.

[33] D. Z. W. Wang and H. K. Lo, "Global optimum of the linearized network design problem with equilibrium flows," Transportation Research Part B: Methodological, vol. 44, no. 4, pp. 482-492, 2010.

[34] P. Luathep, A. Sumalee, W. H. K. Lam, Z. Li, and H. K. Lo, "Global optimization method for mixed transportation network design problem: a mixed-integer linear programming approach," Transportation Research Part B: Methodological, vol. 45, no. 5, pp. 808-827, 2011.

[35] H. Liu and D. Z. W. Wang, "Global optimization method for network design problem with stochastic user equilibrium," Transportation Research Part B: Methodological, vol. 72, pp. 2039, 2015

[36] S. Wang, Q. Meng, and H. Yang, "Global optimization methods for the discrete network design problem," Transportation Research Part B: Methodological, vol. 50, pp. 42-60, 2013.

[37] L. Dimitriou, T. Tsekeris, and A. Stathopoulos, "Genetic computation of road network design and pricing stackelberg games with multi-class users," Lecture Notes in Computer Science (including subseries Lecture Notes in Artificial Intelligence and Lecture Notes in Bioinformatics): Preface, vol. 4974, pp. 669-678, 2008.

[38] G. A. Davis, "Exact local solution of the continuous network design problem via stochastic user equilibrium assignment," Transportation Research Part B: Methodological, vol. 28, no. 1, pp. 61-75, 1994.

[39] Q. Meng, H. Yang, and M. G. H. Bell, "An equivalent continuously differentiable model and a locally convergent algorithm for the continuous network design problem," Transportation Research Part B: Methodological, vol. 35, no. 1, pp. 83-105, 2001.

[40] S.-W. Chiou, "Bilevel programming for the continuous transport network design problem," Transportation Research Part B: Methodological, vol. 39, no. 4, pp. 361-383, 2005.

[41] P. Fontaine and S. Minner, "Benders decomposition for discrete-continuous linear bilevel problems with application to traffic network design," Transportation Research Part B: Methodological, vol. 70, pp. 163-172, 2014.

[42] Q. Meng and X. Wang, "Intermodal hub-and-spoke network design: incorporating multiple stakeholders and multi-type containers," Transportation Research Part B: Methodological, vol. 45, no. 4, pp. 724-742, 2011. 


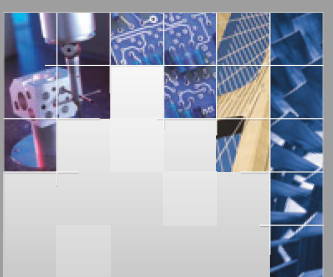

\section{Enfincering}
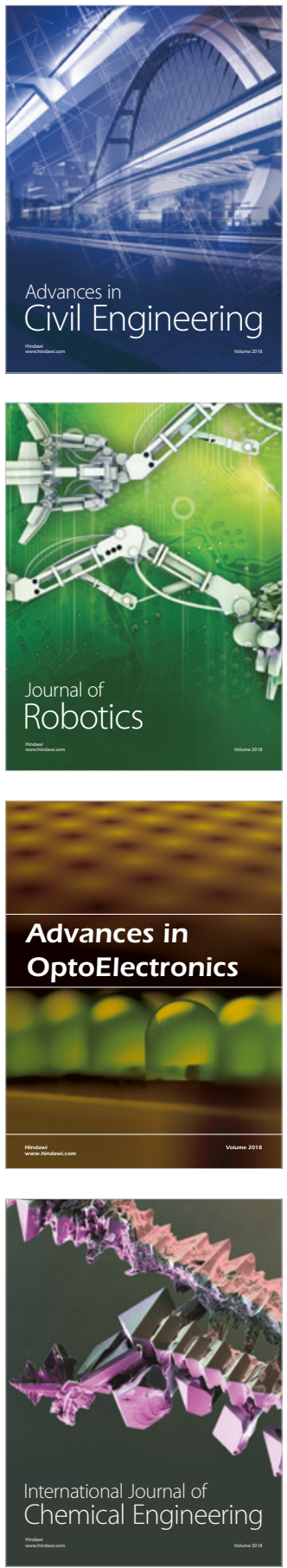

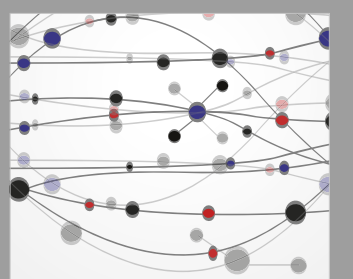

\section{Rotating \\ Machinery}

The Scientific World Journal

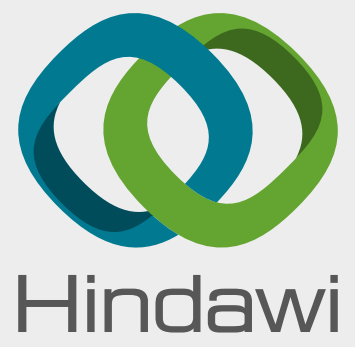

Submit your manuscripts at

www.hindawi.com
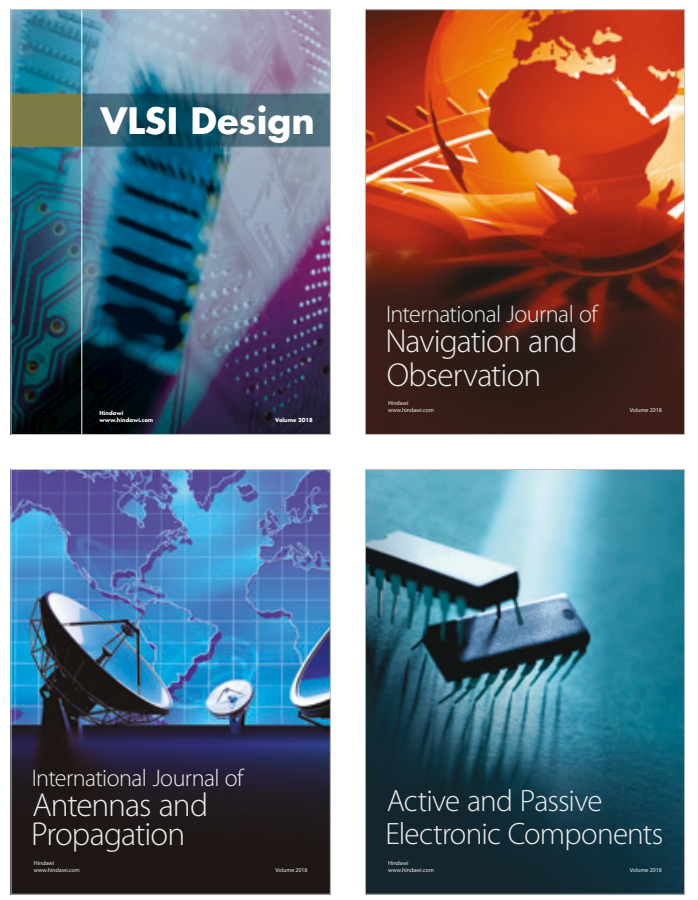
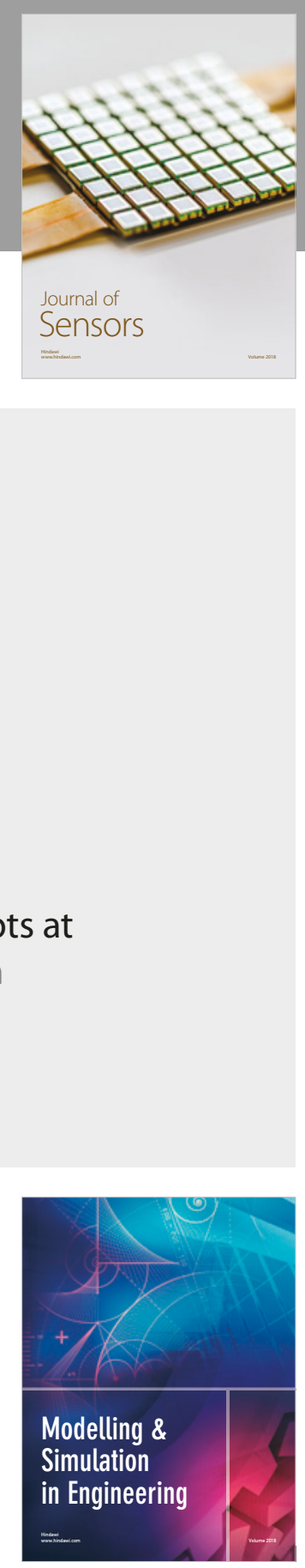

\section{Advances \\ Multimedia}
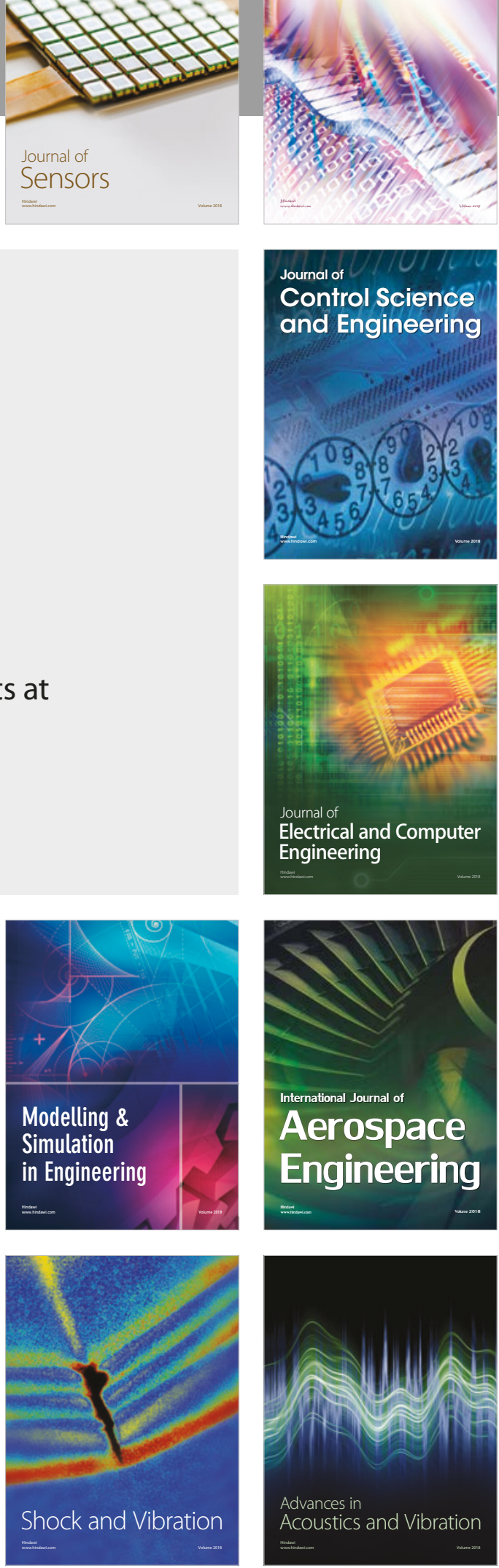